Research Paper

\title{
A Voxel-based Morphometric Analysis of Cerebral Gray Matter in Subcor- tical Ischemic Vascular Dementia Patients and Normal Aged Controls
}

\author{
Chuanming Li', Hanjian Du ${ }^{1 凶}$, Jian Zheng ${ }^{2}$ and Jian Wang1 \\ 1. Department of Radiology, Southwest Hospital, Third Military Medical University, Chongqing, China \\ 2. Department of Neurology, Xinqiao Hospital, Third Military Medical University, Chongqing, China
}

Corresponding author: Hanjian Du, M.D., Department of Radiology, Southwest Hospital, Third Military Medical University, Chongqing, China. Ph: 086-23-68754419; Fax: 086-23-65463026; E-mail: bigcrab551@163.com

(c) Ivyspring International Publisher. This is an open-access article distributed under the terms of the Creative Commons License (http://creativecommons.org/ licenses/by-nc-nd/3.0/). Reproduction is permitted for personal, noncommercial use, provided that the article is in whole, unmodified, and properly cited.

Received: 2011.03.31; Accepted: 2011.07.12; Published: 2011.08.13

\begin{abstract}
BACKGROUND AND PURPOSE: The present study was designed to detect the abnormalities of the cerebral grey-matter density in subcortical ischemic vascular dementia patients by FSL-VBM method to promote the early diagnosis of it.

METHODS: Nine subcortical ischemic vascular dementia patients and nine age-matched normal controls underwent MRI brain structure scanning that was performed on a SIEMENS AVANTO I.5 Tesla scanner and standard TI-weighted high-resolution anatomic scans of MPRAGE sequence were obtained. The 3-demensional MPRAGE images were processed with FSL-VBM package and the cerebral gray matter density was compared between the subcortical ischemic vascular dementia patients and normal controls.

RESULTS: Compared with the normal control group, the cerebral gray matter density of subcortical ischemic vascular dementia patients was found significantly decreasing, including brain regions of thalamus, parietal lobe, frontal lobe and temporal lobe $(P<0.05)$.

CONCLUSIONS: The cerebral gray matter density alterations have closed correlation with cognitive dysfunction in subcortical ischemic vascular dementia patient and can be detected by MRI. MRI has some potential value in the diagnosis of them.
\end{abstract}

Key words: cerebral grey-matter density, subcortical ischemic vascular dementia, MRI

\section{Introduction}

Vascular dementia (VD) is the third most common cause of dementia (8 to $10 \%$ ), following Alzheimer's disease (AD) (60 to $70 \%)$ and dementia with Lewy bodies (DLB) (10 to 25\%), but these numbers vary considerably according to the different criteria used for $\mathrm{VaD}[1,2]$. Subcortical ischemic vascular dementia (SIVD) is an important subtype of them caused by the cerebral vessels factors (ischemia, hypoxia, etc.) which lead to brain tissue damage. It is characterized by lacunar infarcts and deep white matter changes and affects the inner parts of the brain consisting of deep white matter made up of nerve cell fibers. The term "Binswagner's disease" is used when such demyelination is widespread. The syndromes consist of loss of memory, judgment, reasoning, and changes in mood, behavior and communication abilities [3]. The proportion of vascular dementia $(\mathrm{VaD})$ attributed to SIVD ranges from 36 to $50 \%$, with higher rates in African Americans and Asian Americans [4] than whites $[5,6]$.

For AD patients, brain's anatomical structures have been analyzed using the VBM (voxel-based morphometry) technique recently, demonstrating that the gray matter volume of the hippocampus, para- 
hippocampal gyrus, insula, superior/middle temporal gyrus, thalamus, cingulate gyrus, and superior/inferior parietal lobule is decreased. Volumetric analysis of the entorhinal cortex can even distinguish subjects who were destined to develop dementia from normal controls with high accuracy [7]. Recently it has been found subcortical vascular dementia (SIVD) patients has notable cortical thickness and volume alterations in frontal and tempral cortices, and the conversion from $\mathrm{MCI}$ associated with small-vessel disease (svMCI) to SIVD is associated with cortical atrophy in dorsolateral prefrontal and temporal cortices [8-9]. Until now alterations of cortical gray matter density in subjects with cognitive impairment have not been reported. The aim of the present study was to prove the possibility of gray matter density detection using FSL-VBM (http://www.fmrib.ox.ac.uk /fsl/fslvbm) [10-11], to characterize the cerebral morphology and atrophy patterns of the cortex, and to provide a new method and biomarker for the diagnosis of SIVD.

\section{Materials and methods}

\section{Subjects}

A total of 18 right-handed elderly subjects were included in this study. Nine SIVD patients were recruited by physician referral from the Department of Neurology in Southwest Hospital, Chongqing, China (Table 1). The clinical diagnosis of SIVD was made by a senior neurologist according to the criteria suggested by Erkinjuntti [12] and DSM-IV (Diagnostic and Statistical Manual of Mental Disorders, IV). All patients had undergone neurological, neuropsychological and structural neuroimaging evaluation. AD patients with subcortical ischemia or mixed dementia who can also meet DSM-IV VaD criteria and the patients with large territorial infarction involving cortex or single strategic infarction such as anterior nucleus of thalamus were excluded. All patients have visible white matter hyperintensities (WMH) (grade 3 or more by a reported rating scale [13]) addressed with T2/FLAIR imaging data in MRI scanning. Cognitive performance was evaluated through neuropsychological tasks that included: Mini Mental State Examination (MMSE), Clinical Dementia Rating (CDR), Neuropsychiatric Inventory (NPI), Activities of Daily Living Scale (ADL), Hachinski and Hamilton test. Nine age- and sex-matched old people with normal-cognition were recruited from surrounding regions by advertisement. They underwent a comprehensive brain examination to ensure that they had normal brain structure and no neurological lesions. Exclusion criteria included patients with aphasia or who otherwise could not complete psychological testing because of a language disorder; diagnosed chronic or degenerative disease affecting the central nervous system; active substance abuse disorders; intracranial hemorrhage; difficult to control epilepsy that could present with cognitive impairment; or temporal lobe epilepsy. All of those subjects who with brain trauma, brain tumor, psychiatric disorders, systemic disease or other MRI contraindication such as claustrophobia were excluded from this study. Present or past use of medication to treat cognition and infarction was not an exclusion criterion. All subjects provided informed consents and the ethics committee at the Third Military Medical University approved the study protocols.

\section{MRI data acquisition}

The MRI brain scanning was performed on a SIEMENS AVANTO 1.5 Tesla scanner with head matrix coil at the Department of Radiology in Southwest Hospital, Chongqing, China. During the examination, each subject laid supine in the scanner with eyes closed. Scanner noise was attenuated with earplugs and head motion was restricted with foam padding (provided by the manufacturer) around the head and the necessity of head immobility was emphasized to each subject. Anatomical image datasets were acquired with a standard T1-weighted high-resolution anatomic scan of magnetization-prepared rapid gradient echo (MPRAGE) sequence: TR $=1900 \mathrm{~ms}$, $\mathrm{TE}=3.37 \mathrm{~ms}, \mathrm{TI}=1100 \mathrm{~ms}$, flip angle $=15^{\circ}, \mathrm{FOV}=256 \mathrm{~mm}$ $\times 192 \mathrm{~mm}, \mathrm{NEX}=1$, matrix $=256 \times 192,1.00 \times 1.00 \mathrm{~mm}^{2}$ in-plane resolution, horizontal slices with slice thickness of $1.3 \mathrm{~mm}$ and no gap. Acquisition time was $3.55 \mathrm{~min}$. The MPRAGE acquisition of each study was ensured the same slice orientation (paralleled to the anterior commissure (AC) and commissural posterior (PC) line). Subsequently, T2-weighted (T2WI) images with 36 axial slices, $3 \mathrm{~mm}$ thick and $1 \mathrm{~mm}$ gap, oriented to AC-PC line were obtained.

\section{MRI data analysis}

Before further analysis of the 3D brain images, we confirmed that all images were uncontaminated with major head motion. The $3 \mathrm{D}$ data were copied from the MRI scanner and imported to a personal computer running a Linux operating system, on which off-line analysis was carried out by anatomical structure image analysis software FSL-VBM.

First, by using mri_convert command, DICOM files were completely converted to nii.gz format and then these structural images were brain-extracted using BET [14] in order to remove skin and skull. Next, tissue-type segmentation was carried out using 
FAST4 [15] package, after that gray matter, white matter and CSF were produced. The resulting gray-matter partial volume images were then aligned to MNI152 standard space using the affine registration tool FLIRT $[16,17]$, followed optionally by nonlinear registration using FNIRT, which uses a b-spline representation of the registration warp field [18]. The resulting images were averaged to create a study-specific template in order to reduce the effect of inter-subject brain variability during the registration procedure, to which the native gray matter images were then non-linearly re-registered. The registered partial volume images were modulated (to correct for local expansion or contraction) by dividing using the Jacobian of the warp field. The modulated segmented images were then smoothed with an isotropic Gaussian kernel with a sigma of $3 \mathrm{~mm}$ for the TFCE-based analysis [19]. Finally, differences in cerebral gray matter density between the patient group and control group were assessed by voxelwise GLM applied using permutation-based non-parametric testing (5000 permutations) [20]. Cluster-size shareholding at p-values $<0.05$, fully correcting for multiple comparisons across space, was used.

\section{Results}

The two groups did not differ significantly in age, sex ratio, or education. The scores include MMSE, NPI, CDR and ADL, as well as those key excluded measuring scales such as Hachinski score and Hamilton score were summarized in Table 1.

The cerebral gray matter density in SIVD patients were found significant decreasing than that of the normal controls in brain regions of thalamus, parietal lobe, frontal lobe and temporal lobe $(P<0.05)$. (Table 2, Figure 1)

Table I. Characteristics of the study participants [mean (S.D.)]

\begin{tabular}{|c|c|c|c|c|}
\hline Groups & SIVD & Controls & $\mathrm{X}^{2} / \mathrm{T}$ & $\mathrm{p}$-value \\
\hline $\mathrm{N}$ & 9 & 9 & & \\
\hline Age & $62.3(6.1)$ & $60.4(4.2)$ & 2.47 & $\mathrm{P}>0.05$ \\
\hline Female/male & $4 / 5$ & $4 / 5$ & 1.72 & $\mathrm{P}>0.05$ \\
\hline Education (years) & $6.2(2.1)$ & $6.5(4.0)$ & 2.44 & $\mathrm{P}>0.05$ \\
\hline MMSE & $15.4(3.6)$ & $28.3(2.4)$ & 19.56 & $\mathrm{P}<0.01$ \\
\hline NPI & $26.20(10.3)$ & & & \\
\hline $\mathrm{ADL}$ & $38.5(7.2)$ & & & \\
\hline Hachinski & $12.5(3.4)$ & & & \\
\hline Hamilton & $9.7(3.2)$ & & & \\
\hline
\end{tabular}

Table 2. Local maximums of the significant clusters showing decreased cerebral gray matter density in SIVD when compared with the controls $(P<0.05)$.

\begin{tabular}{|c|c|c|c|c|c|}
\hline Cerebral gray matter & Hemisphere & Z-MAX & $\mathrm{MN}$ & & \\
\hline & & & $x$ & $\mathrm{y}$ & $\mathrm{z}$ \\
\hline Thalamus & $\mathrm{R}$ & 1 & 4 & -14 & -8 \\
\hline Parietal lobe & $\mathrm{R}$ & 0.992 & 36 & -46 & 40 \\
\hline Frontal lobe & $\mathrm{R}$ & 0.998 & 48 & 6 & -4 \\
\hline Frontal lobe & $\mathrm{L}$ & 0.996 & -40 & 48 & 26 \\
\hline Occipital lobe & $\mathrm{L}$ & 0.96 & -46 & -74 & -4 \\
\hline Frontal lobe & $\mathrm{L}$ & 0.959 & -36 & 42 & 10 \\
\hline Parietal lobe & $\mathrm{R}$ & 0.958 & 34 & -80 & 30 \\
\hline Parietal lobe & $\mathrm{L}$ & 0.964 & -34 & -72 & 52 \\
\hline Occipital lobe & $\mathrm{L}$ & 0.967 & 8 & -80 & 4 \\
\hline Frontal lobe & $\mathrm{L}$ & 0.957 & 46 & 30 & 28 \\
\hline Temporal lobe & $\mathrm{L}$ & 0.996 & 68 & -4 & -8 \\
\hline Frontal lobe & $\mathrm{L}$ & 0.953 & 52 & 22 & 40 \\
\hline
\end{tabular}




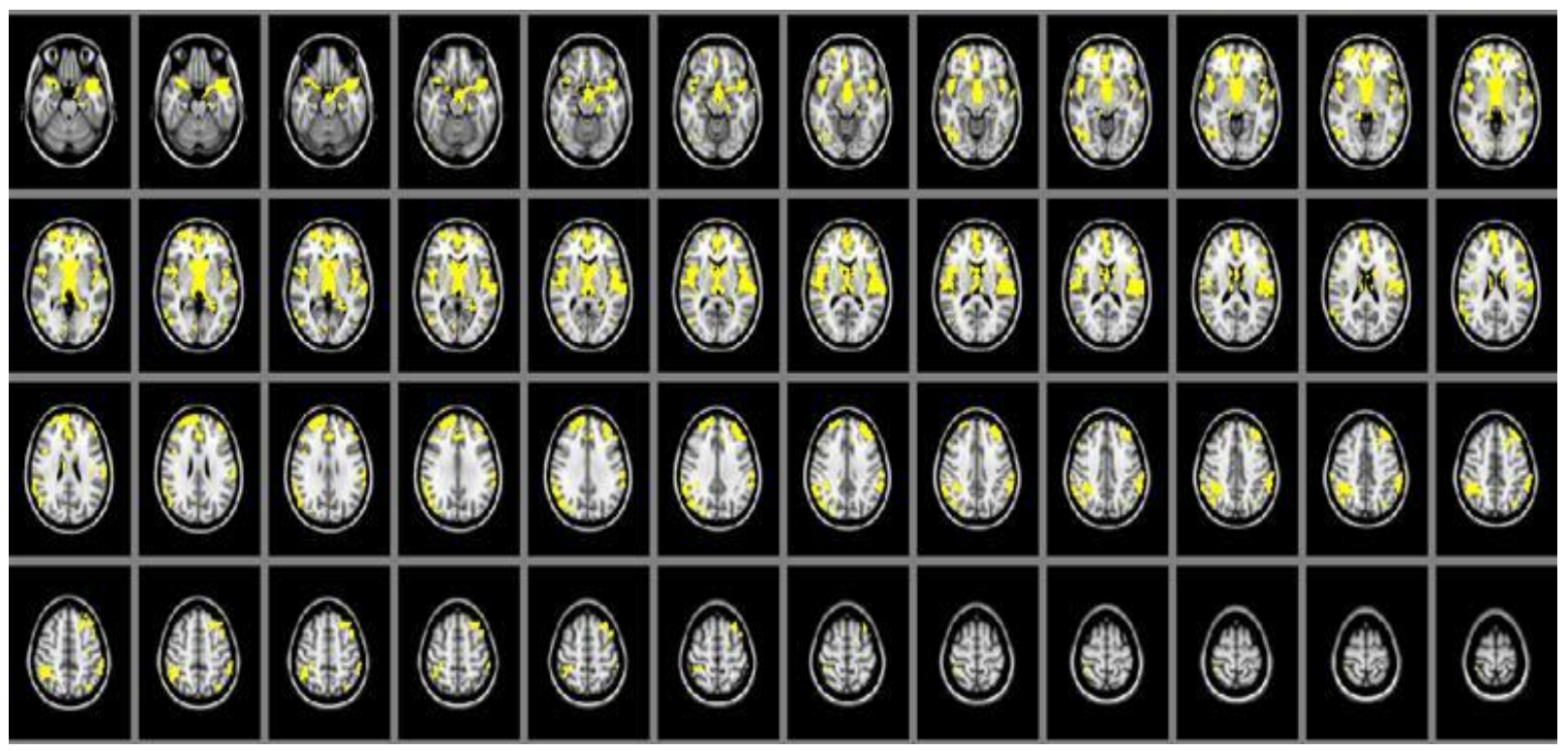

Figure. I. Area of cerebral gray matter density alteration in SIVD patients detected by MRI is shown in yellow color, including thalamus, parietal lobe, frontal lobe and temporal lobe, compared with control group.

\section{Discussion}

To date, limited studies of cortical gray matter density have been performed due to the lack of efficient methods. Before the methods for automatic calculation of cortical thickness were established, measurement of cortical thickness was performed primarily on the basis of manual contouring, which inevitably incurred significant inter-operator variability. Manual measurement also has the drawback of inability to achieve sub-millimeter precision; therefore the accuracy of the measurements is quite questionable.

FSL-VBM is more accurate than SPM-VBM and can found more significant cluster [10], because this method depends on the degrees of freedom of the non-rigid registration [21] and non-linear registration The alteration in cortical thickness have been well studied in several neurological diseases, such as multiple sclerosis [11], schizophrenia [10] and so on, by using the FSL-VBM analysis. SvMCI patients have been reported cortical thinning in inferior frontal and orbitofrontal gyri, anterior cingulate, insula, superior temporal gyrus, and lingual gyrus, while cortical thinning in SIVD patients involved all these areas plus dorsolateral prefrontal and temporal cortices [22]. To our best knowledge, the present study represents the first attempt to adapt this method to analyze the cerebral gray matter density of vascular cognitive impairment. In this study, the MRI technique and anaphase analysis method provide a new way to study the structure of the brain. Gray matter density in brain regions of thalamus, parietal lobe, frontal lobe and temporal lobe were found decreasing greatly, which MRI can detect. These findings suggest that these brain regions may be the most affected in SIVD, that the alterations in gray matter density are a possible characteristic of this type of dementia, and that the measurement of gray matter density in the above regions might help its detection. Temporal lobe has been found closed correlation with memory and emotion. Based on experimental evidence from both monkeys as well as humans, the prefrontal cortex has been proved to be important not only for attention, emotion and execution processing, but also for inhibition of "prepotent" habitual [23]. Some human diseases, such as schizophrenia, involving prefrontal abnormalities result in impairments in attention conflict paradigms [24]. The parietal lobes play an important role in word processing. For example, the region of the left inferior parietal has been reported to become active when processing words compared to letter strings. The lateral left inferior region has been reported to be more active when viewing or naming words compared to pictures [25]. Thalamus lesion was found associate with memory damage in animal [26] and other human studies [27]. Previously, by using Tc-HMPAO SPECT method, regional cerebral blood flow in thalamus was found decreased significant in vascular dementia when compared with that in the controls [28].

Vascular cognitive impairment may affect half of all stroke survivors [29]. There is no cure for vascular dementia until now and the focus is currently on early 
diagnosis and preventing further brain damage. Diagnostic research criteria for SIVD are based on magnetic resonance imaging (MRI) findings including substantial white matter lesions (WML), multiple lacunar infarcts, and neuropsychological tests. Efforts to relate MRI-measured lacunes and white matter changes to cognitive impairment have not been straightforward $[26,30]$. In this study we found a new biological marker of SIVD patients, which can be detected conveniently by FSL -VBM. As a new atraumatic technology, we believe FSL -VBM has much potential value and could promote the diagnosis of SIVD in future.

\section{Acknowledgments}

We thank Mark Jenkinson for assistance in data analysis. This study was supported by the National Natural Science Foundation of China (Grant No. 81000607).

\section{Conflict of Interest}

The authors have declared that no conflict of interest exists.

\section{References}

1. Jellinger KA. The enigma of vascular cognitive disorder and vascular dementia. Acta Neuropathol. 2007; 113: 349-88.

2. Pohjasvaara T, Mantyla R, Ylikoski R, et al. Comparison of different clinical criteria (DSM-III, ADDTC, ICD-10, NINDS-AIREN, DSM-IV) for the diagnosis of vascular dementia. National Institute of Neurological Disorders and Stroke-Association Internationale pour la Recherche et l'Enseignement en Neurosciences. Stroke. 2000; 31: 2952-7.

3. Riekse RG, Leverenz JB, McCormick W, et al. Effect of vascular lesions on cognition in Alzheimer's disease: a community-based study. J Am Geriatr Soc. 2004; 52: 1442-8.

4. Ross GW, Petrovitch H, White LR, et al. Characterization of risk factors for vascular dementia: The Honolulu Asia Aging Study. Neurology. 1999; 53: 337-43.

5. Loeb C, Gandolfo C, Croce R, et al. Dementia associated with lacunar infarction. Stroke. 1992; 23:1225-9.

6. Tatemichi TK, Desmond DW, Paik M, et al. Clinical determinants of dementia related to stroke. Ann Neurol. 1993; 33: 568-75.

7. Xiaojuan G, Zhiqun W, Kuncheng L, et al. Voxel-based assessment of gray and white matter volumes in Alzheimer's disease. Neuroscience Letters. 2010; 468: 146-50.

8. Jagust WJ, et al. Neuropathological basis of magnetic resonance images in aging and dementia. Ann Neurol. 2008 Jan;63(1):72-80.

9. Seo SW. Cortical thinning in vascular mild cognitive impairment and vascular dementia of subcortical type. J Neuroimaging. 2010 Jan;20(1):37-45.

10. Douaud G, Smith S, Jenkinson M, et al. Anatomically related grey and white matter abnormalities in adolescent-onset schizophrenia. Brain. 2007; 130: 2375-86.

11. Battaglini M, Giorgio A, Stromillo ML, et al. Voxel-wise assessment of progression of regional brain atrophy in relapsing-remitting multiple sclerosis. J Neurol Sci. 2009; 282:55-60.
12. Erikinjuntti $T$, Inzitari $D$, Pantoni $L$, et al. Research criteria for subcortical vascular dementia in clinical trials. Neural Transm Suppl. 2000; 59: 23-30.

13. Fazekas F, Chawluk A, Alavi HI, et al. MR signal abnormalities at $1.5 \mathrm{~T}$ in Alzheimer's dementia and normal aging. American Journal of Roentgenology. 1987; 149: 351-6.

14. Smith SM. Fast robust automated brain extraction. Hum Brain Mapp. 2002; 17: 143-55.

15. Zhang Y, Brady M, Smith S. Segmentation of brain MR images through a hidden Markov random field model and the expectation-maximization algorithm. IEEE Trans Med Imaging. 2001; 20: 45-57.

16. Jenkinson $M$, Bannister $P$, Brady $M$, et al. Improved optimization for the robust and accurate linear registration and motion correction of brain images. Neuroimage. 2002; 17: 825-41.

17. Jenkinson M, Smith S. A global optimisation method for robust affine registration of brain images. Med Image Anal. 2001; 5: $143-56$.

18. Rueckert D, Sonoda LI, Hayes C, et al. Nonrigid registration using free-form deformations: application to breast MR images. IEEE Trans Med Imaging. 1999; 18: 712-21.

19. Smith SM, Nichols TE. Threshold-free cluster enhancement: addressing problems of smoothing, threshold dependence and localisation in cluster inference. Neuroimage. 2009; 44: 83-98.

20. Nichols TE, Holmes AP. Nonparametric permutation tests for functional neuroimaging: a primer with examples. Hum Brain Mapp. 2002; 15: 1-25.

21. Crum WR, Griffin LD, Hill DL, et al. Zen and the art of medical image registration: correspondence, homology, and quality. Neuroimage. 2003; 20: 1425-37.

22. Seo SW, Ahn J, Yoon U, et al. Cortical thinning in vascular mild cognitive impairment and vascular dementia of subcortical type. J Neuroimaging. 2010; 20: 37-45.

23. Frith $C$, Dolan R. The role of the prefrontal cortex in higher cognitive functions. Cog Brain Res. 1996; 5: 175-81.

24. Yucel M, Pantelis C, Stuart GW, et al. Anterior cingulate activation during Stroop task performance: a PET to MRI coregistration study of individual patients with schizophrenia. Am-J-Psychiatry. 2002; 159: 251-4.

25. Jessen F, Erb M, Klose U, et al. Activation of human language processing brain regions after the presentation of random letter strings demonstrated within event-related functional magnetic resonance imaging. Neuroscience Letters. 1999; 270: 13-6.

26. Aggleton JP, Neave N, Nagle S, et al. A comparison of the effects of anterior thalamic, mamillary body and fornix lesions on reinforced spatial alternation. Behav Brain Res. 1995; 68: 91-101.

27. Akiguchi I, Ino T, Nabatame $\mathrm{H}$, et al. Acute-onset amnestic syndrome with localized infarct on the dominant side--comparison between anteromedial thalamic lesion and posterior cerebral artery territory lesion. Jpn J Med. 1987; 26: 15-20.

28. Gold G, Kovari E, Herrmann FR, et al. Cognitive consequences of thalamic, basal ganglia, and deep white matter lacunes in brain aging and dementia. Stroke. 2005; 36: 1184-8.

29. Tatemichi TK, Paik M, Bagiella E, et al. Risk of dementia after stroke in a hospitalized cohort: results of a longitudinal study. Neurology. 1994; 44: 1885-91.

30. O'Sullivan M, Morris RG, Huckstep B, et al. Diffusion tensor MRI correlates with executive dysfunction in patients with ischaemic leukaraiosis. J Neurol Neurosurg Psychiatry. 2004; 75: 441- 7 . 